\title{
Effectiveness and Safety of Aliskiren and Aliskiren Hydrochlorothiazide (HCT) in a Multiethnic, Real-World Setting
}

Srinivasa Rao Maddury · Abhay Pande - K. M. H. S. Sirajul Haque · Akram Echtay · Loewe Go · Tashbeeb Gulzar · Mahomed Kadwa · Sashka Hristoskova

To view enhanced content go to www.advancesintherapy.com Received: November 16, 2012 / Published online: January 25, 2013

(c) Springer Healthcare 2013

\section{ABSTRACT}

Introduction: Numerous randomized clinical trials have demonstrated the efficacy and tolerability of aliskiren and aliskiren

S. R. Maddury $(\bowtie)$

CARE Hospital, D.No.8-2-330/A/2, Banjara Hills, Road No. 5, Opposite Nokia Towers, Hyderabad 500 034, India

e-mail: maddurysrinivas@hotmail.com

A. Pande

Al Zahra Hospital, Sharjah, UAE

K. M. H. S. S. Haque

Anwer Khan Modern Medical College, Dhanmondi, Dhaka

A. Echtay

Hariri University Hospital, Beirut, Lebanon

L. Go

Saint Luke's Medical Center, Quezon City, Philippines

T. Gulzar

Hameed Latif Hospital, Lahore and the National

Hospital Lahore, Pakistan

M. Kadwa · S. Hristoskova

Novartis Pharma AG, Basel, Switzerland

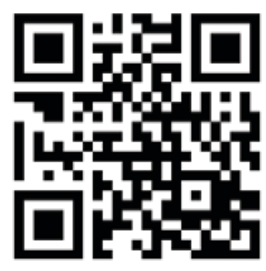

Enhanced content for Advances in Therapy articles is available on the journal web site: www.advancesintherapy.com hydrochlorothiazide (aliskiren HCT) singlepill combination therapy in patients with hypertension. The objective of the present study was to evaluate the effectiveness and safety of aliskiren-based therapy under daily life conditions in a multiethnic population.

Methods: This observational, multicenter, noninterventional study, conducted at 420 centers in Asia and the Middle East, included adult patients with hypertension who received treatment with aliskiren or aliskiren HCT as single or add-on therapy for a planned treatment period of at least 26 weeks. The main effectiveness assessments included the proportion of patients achieving therapeutic blood pressure (BP) goal (defined as systolic BP [SBP]/diastolic BP [DBP] $<140 / 90 \mathrm{mmHg}$, or $<130 / 80 \mathrm{mmHg}$ in patients with diabetes) and $\mathrm{BP}$ response, and change in mean sitting BP from baseline to study end.

Results: Of 4,826 patients (mean age 51.4 years, $65.9 \%$ male, $64.5 \%$ Asian, $41.5 \%$ diabetic) included in the study, 3,473 received aliskiren and 1,353 received aliskiren HCT. Almost half the study population $(48.1 \%)$ received aliskiren or aliskiren HCT as add-on therapy. The therapeutic BP goal was achieved in $49.5 \%$ of patients treated with aliskiren and $48.3 \%$ of patients 
receiving aliskiren HCT; attainment of BP goal increased to more than $70 \%$ when a classic BP target of $<140 / 90 \mathrm{mmHg}$ was applied for all patients. Reductions in mean sitting SBP/DBP were significantly lower versus baseline for both aliskiren (24.1/12.2 $\mathrm{mmHg})$ and aliskiren HCT $(27.6 / 14.1 \mathrm{mmHg})$ and $\mathrm{BP}$ response rates were consistently achieved in more than $80 \%$ of all patients during the study. Aliskiren treatment was well tolerated with only a small proportion of patients experiencing adverse events (AEs; $2.1 \%$ ) and serious AEs (0.3\%).

Conclusion: In this real-world, naturalistic setting, antihypertensive treatment with an aliskiren-based regimen was effective and welltolerated in this multiethnic population with arterial hypertension.

Keywords: Aliskiren; Aliskiren HCT; Asia; Direct renin inhibitor; Effectiveness; Hypertension; Middle East; Observational; Real-world; Tolerability

\section{INTRODUCTION}

As the number one cause of mortality worldwide [1], there is no doubt that hypertension is a major public health concern in today's society. Current statistics report that globally almost 1 billion individuals have high blood pressure (BP) [2, 3], and by 2025 , the prevalence of hypertension is expected to increase by about $60 \%$ to 1.56 billion [2]. Importantly, almost threequarters of this hypertensive population will reside in economically developing countries [2].

Hypertension is one of the most common and treatable risk factors for cardiovascular (CV) morbidity and mortality [3]. It is also often clustered with other CV risk factors such as diabetes, obesity, and metabolic syndrome, placing patients at even greater risk of $\mathrm{CV}$ disease [4, 5]. Data from 2001 showed that nonoptimal BP, defined as systolic BP (SBP) $\geq 115 \mathrm{mmHg}$, was responsible for $54 \%$ of all strokes and $47 \%$ of all cases of ischemic heart disease [6]. Moreover, approximately $80 \%$ of the $\mathrm{CV}$ disease burden attributable to hypertension occurred in low- and middle-income countries, including Asia and the Middle East [6].

Despite evidence showing that effective BP lowering can reduce the risk of CV events [7], and the availability of numerous efficacious antihypertensive agents, control of high BP to guideline-recommended targets remains a global challenge $[8,9]$. Indeed, in parts of Asia, BP control rates in patients receiving antihypertensive treatment are as low as $20 \%$ [10], a reflection of what is being seen elsewhere in the world. There are numerous reasons for failure to achieve BP targets in patients receiving antihypertensive treatment, including noncompliance/nonpersistence with therapy, suboptimal antihypertensive treatment efficacy, and poorly tolerated regimens [11].

Aliskiren is a first-in-class direct renin inhibitor, which acts at the rate-limiting step of the renin-angiotensin-aldosterone system (RAAS) and reduces plasma renin activity, angiotensin I, angiotensin II, and aldosterone [12, 13]. Numerous randomized, controlled clinical trials have demonstrated the efficacy and tolerability of aliskiren and the combination of aliskiren hydrochlorothiazide (aliskiren HCT) across a range of hypertensive patient populations, including those deemed difficult to treat, such as patients with stage 2 hypertension [14], metabolic comorbidities, or obesity [15, 16], and the elderly [17].

As only limited published data are available on the real-world effectiveness and safety of aliskiren [18-20], particularly in regions such as Asia and the Middle East, the present study was conducted to characterize and assess the 
effectiveness, safety and tolerability of aliskiren and the single-pill combination (SPC) of aliskiren HCT as antihypertensive therapy under dailylife conditions during a planned observation and treatment period of at least 26 weeks in a multiethnic population.

\section{MATERIALS AND METHODS}

\section{Study Design and Patient Population}

This was an observational, multicenter, prospective, open-label, single-arm study conducted at 420 centers in Asia (Bangladesh, India, the Philippines, and Pakistan) and the Middle East (Bahrain, Kuwait, Lebanon, Oman, Qatar, and the United Arab Emirates) between June 2010 and August 2011. The study was conducted as a noninterventional trial and as such, therapy was prescribed in terms of the marketing authorization in the respective countries; the assignment of the patient to therapy was as per usual practice and clinically indicated, and was clearly separated from the decision to include the patient in the study. No additional diagnostic or monitoring procedures other than those usually performed as part of routine patient care were applied to the patients. The study protocol recommended that BP measurements were made in accordance with the 1988 American Heart Association Committee Report on BP determination [21]; however, this was an observational study and recommendations were made for guidance purposes only.

Male and female patients aged $\geq 18$ years with an established diagnosis of hypertension for which treatment with aliskiren or aliskiren HCT SPC as single or add-on therapy had been prescribed by a treating physician were eligible for inclusion in the study. Patients with any contraindication (as stated in the local prescribing information for the respective participating country) to aliskiren or aliskiren HCT were excluded from participation. Informed written consent for the collection and use of data was obtained from all participants, and the study was performed in accordance with national requirements and regulations. In accordance with the observational nature of this study, patients were not required to consent to taking the medication of interest, as treatment was decided prior to study entry in line with usual clinical practice. Patients were discontinued from the study if they withdrew informed consent or were no longer taking the medication of interest.

During the observational period of $26 \pm 8$ weeks, data from at least two routine patient visits were recorded: the baseline visit, during which inclusion and exclusion criteria were assessed and baseline data collected, and a final visit at the end of the study. Additionally, the treating physician could record data from a visit performed at approximately 13 weeks after initiation of the study. Data collected included patient demographics, patient characteristics, details of the antihypertensive therapies prescribed, including dose of aliskiren or aliskiren HCT, concomitant medications, vital signs and weight measurements, laboratory values (if conducted), adverse events (AEs), and a final investigator assessment of the therapy with regard to effectiveness, tolerability and compliance.

\section{Outcomes Assessments}

The main effectiveness criterion was the proportion of patients who achieved therapeutic goal, defined as a target SBP $<140 \mathrm{mmHg}$ and diastolic BP (DBP) $<90 \mathrm{mmHg}$. For patients with comorbid diabetes, the respective SBP and DBP goals were $<130 \mathrm{mmHg}$ and $<80 \mathrm{mmHg}$ at 
the end of the study. Additional effectiveness criteria included the absolute change from baseline to end of study in mean sitting SBP (msSBP) and mean sitting DBP (msDBP), and the proportion of patients achieving a BP response (SBP response defined as SBP $<140 \mathrm{mmHg}$ [ $<130 \mathrm{mmHg}$ in patients with diabetes] or a reduction of $\geq 20 \mathrm{mmHg}$; DBP response defined as DBP $<90 \mathrm{mmHg}$ [ $<80 \mathrm{mmHg}$ in patients with diabetes] or a reduction of $\geq 10 \mathrm{mmHg}$ ). Safety and tolerability were assessed by monitoring and recording all AEs, serious AEs (SAEs), laboratory safety evaluations (if performed), physical examinations, vital signs, and body weight. At final study visit, an additional assessment was performed relating to the effectiveness and tolerability of therapy with aliskiren or aliskiren HCT, as well as treatment compliance, as judged by the study investigator according to the following grading: "below average," "average," "good," or "very good."

\section{Statistical Analyses}

Data from all countries and sites were pooled. All effectiveness and safety analyses were performed on the full analysis set (FAS), consisting of patients who provided informed consent, were assigned a patient number, and entered the study. Demographics and baseline characteristics were summarized with standard descriptive statistics. The proportion of patients achieving the therapeutic goal and BP response were summarized by visit. If no BP measurements were available at week 26, the week 13 measurement was carried forward (last observation carried forward $[\mathrm{LOCF}])$. Changes from baseline in SBP and DBP were summarized with standard descriptive statistics by visit and analyzed with a one-sided $t$-test examining the hypothesis of no decrease from baseline. The test results were accompanied by $95 \%$ two-sided confidence intervals (CIs) for the mean. An additional analysis was performed for therapeutic BP goal and BP response using a classic BP target for patients with hypertension, i.e., $<140 / 90 \mathrm{mmHg}$ for therapeutic goal, $<140 \mathrm{mmHg}$ (or a reduction in SBP of $\geq 20 \mathrm{mmHg}$ ) for SBP response; and $<90 \mathrm{mmHg}$ (or a reduction in DBP of $\geq 10 \mathrm{mmHg}$ ) for DBP response. Data analysis was performed by DATAMAP GmbH, Freiburg, Germany using SAS, version 9.1 (SAS Institute, Cary, NC, USA).

\section{RESULTS}

\section{Patient Disposition}

Of the 5,366 patients enrolled in the study, evaluable data were available for 4,829 patients, all of whom were included in the FAS. Of these, three patients were excluded from the analyses because antihypertensive treatment was unknown. In total, 3,473 patients were treated with aliskiren (150 mg, $n=2,183 ; 300 \mathrm{mg}$, $n=1,290)$, while 1,353 patients were treated with aliskiren HCT SPC $(150 / 12.5 \mathrm{mg}, n=762$; $150 / 25 \mathrm{mg}, n=60 ; 300 / 12.5 \mathrm{mg}, n=452$; $300 / 25 \mathrm{mg}, n=79)$. Both aliskiren and aliskiren HCT were given as single or add-on therapy. The study was completed by 4,600 (95.3\%) patients ( $n=3,278$ in the aliskiren treatment group and $n=1,322$ in the aliskiren HCT group). The main reason for premature discontinuation from the study was loss to follow-up in 181 (3.8\%) patients (Table 1).

All patients included in the study were from Asia (Bangladesh 5.7\%; India 17.9\%; Pakistan 6.2\%; and the Philippines 11.3\%) or the Middle East (Lebanon 22.1\%; United Arab Emirates 23.6\%; Oman 2.8\%; Qatar 3.1\%; Kuwait 4.6\%; and Bahrain 2.7\%). Patient demographics and baseline characteristics are outlined in Table 2. The mean age was 51.4 years and the majority of patients were male $(65.9 \%)$ and 
Table 1 Patient disposition

\begin{tabular}{llll}
\hline & Aliskiren & Aliskiren HCT & Total \\
$n=3,473$ & $n=1,353$ & $n(\%)$ & $n(\%)$ \\
& $n(\%)$ & $1,322(97.7)$ & $4,600(95.3)$ \\
\hline Completed & $3,278(94.4)$ & $30(2.2)$ & $224(4.6)$ \\
Discontinued & $194(5.6)$ & $1(0.1)$ & $2(0.0)$ \\
Unknown & $1(0.0)$ & & $15(0.3)$ \\
Primary reason for premature discontinuation & & $1(0.1)$ & $4(0.1)$ \\
Adverse event(s) & $14(0.4)$ & $0(0.0)$ & $0(0.0)$ \\
Abnormal laboratory value(s) & $4(0.1)$ & $0(0.0)$ & $6(0.1)$ \\
Abnormal test procedure & $0(0.0)$ & $0(0.0)$ & $3(0.1)$ \\
Unsatisfactory therapeutic effect & $6(0.2)$ & $0(0.0)$ & $4(0.1)$ \\
No longer requiring medication of interest & $3(0.1)$ & $0(0.0)$ & $181(3.8)$ \\
Withdrew consent & $4(0.1)$ & $25(1.9)$ & $2(0.0)$ \\
Lost to follow-up & $156(4.5)$ & $0(0.0)$ & $4(0.1)$ \\
Administrative problems & $2(0.1)$ & $1(0.1)$ & $3(0.1)$ \\
Death & $3(0.1)$ & $1(0.1)$ & $2(0.0)$ \\
Protocol deviation & $2(0.1)$ & $2(0.2)$ & $n$ \\
Unknown & $0(0.0)$ & & $n$ \\
\hline Full analysis set & & & \\
\end{tabular}

Full analysis set

HCT hydrochlorothiazide

Table 2 Patient demographics and baseline clinical characteristics

\begin{tabular}{|c|c|c|c|}
\hline Variable & $\begin{array}{l}\text { Aliskiren } \\
(n=3,473)\end{array}$ & $\begin{array}{l}\text { Aliskiren HCT } \\
(n=1,353)\end{array}$ & $\begin{array}{l}\text { Total } \\
(n=4,826)\end{array}$ \\
\hline Age (years), mean $\pm S D$ & $51.0 \pm 11.56$ & $52.6 \pm 10.48$ & $51.4 \pm 11.29$ \\
\hline \multicolumn{4}{|l|}{ Gender, $n(\%)$} \\
\hline Male & $2,294(66.1)$ & $885(65.4)$ & $3,179(65.9)$ \\
\hline Female & $1,178(33.9)$ & $468(34.6)$ & $1,646(34.1)$ \\
\hline \multicolumn{4}{|l|}{ Race, $n(\%)$} \\
\hline Caucasian & $754(21.7)$ & $412(30.5)$ & $1,166(24.2)$ \\
\hline Black & $64(1.8)$ & $29(2.1)$ & $93(1.9)$ \\
\hline Asian & $2,317(66.7)$ & $795(58.8)$ & $3,112(64.5)$ \\
\hline Other & $330(9.6)$ & $115(8.5)$ & $445(9.2)$ \\
\hline $\mathrm{BMI} \geq 30 \mathrm{~kg} / \mathrm{m}^{2}, n(\%)$ & $1,014(29.2)$ & $466(34.4)$ & $1,480(30.7)$ \\
\hline Diabetes, $n(\%)$ & $1,362(39.2)$ & $643(47.5)$ & $2,005(41.5)$ \\
\hline Prior essential hypertension, $n(\%)$ & $3,422(98.5)$ & $1,343(99.3)$ & $4,765(98.7)$ \\
\hline Mean duration of hypertension, $n$ (years) & $1,631(3.28)$ & $626(3.58)$ & $2,257(3.36)$ \\
\hline Prior antihypertensive treatment, $n(\%)^{a}$ & $2,443(70.3)$ & $1,011(74.7)$ & $3,454(71.6)$ \\
\hline \multicolumn{4}{|l|}{ Baseline BP $(\mathrm{mmHg})$, mean $\pm \mathrm{SD}$} \\
\hline SBP & $153.9 \pm 15.01$ & $157.1 \pm 14.98$ & $154.8 \pm 15.07$ \\
\hline DBP & $93.5 \pm 8.94$ & $95.0 \pm 8.76$ & $93.9 \pm 8.92$ \\
\hline
\end{tabular}

Full analysis set

$B M I$ body mass index, $B P$ blood pressure, DBP diastolic blood pressure, HCT hydrochlorothiazide, SBP systolic blood pressure, $S D$ standard deviation

${ }^{a}$ Most frequently used agents prior to the start of the study included angiotensin receptor blockers (26.7\%), beta-blockers (26.5\%) and calcium channel blockers $(26.4 \%)$ 
Asian (64.5\%). Furthermore, the mean duration of hypertension was 3.4 years. Of note, $41.5 \%$ of patients had concomitant diabetes and more than 30\% were classified as being obese (body mass index $[\mathrm{BMI}] \geq 30 \mathrm{~kg} / \mathrm{m}^{2}$ ). Prior to the start of the study, more than $70 \%$ of patients had been treated with antihypertensive medications (predominantly angiotensin II receptor blockers [ARBs], beta-blockers, calcium channel blockers [CCBs], and angiotensin converting enzyme inhibitors [ACEIs]), receiving on average 1.1 antihypertensive medications. In almost half of the patients $(n=2,323 ; 48.1 \%)$, aliskiren $(n=$ $1,685 ; 48.5 \%)$ or aliskiren HCT $(n=638 ; 47.2 \%)$ was prescribed as add-on therapy, with an average of 0.7 different types of antihypertensive medications prescribed per patient in addition to the medication of interest. The most common types of antihypertensive medications used in combination with aliskiren or aliskiren HCT were beta-blockers (20.6\%), CCBs (19.2\%), ARBs (15.5\%), and ACEIs (6.9\%).

\section{Treatment Effectiveness}

The proportion of patients who reached the defined therapeutic BP goal (SBP $<140 \mathrm{mmHg}$ and DBP $<90 \mathrm{mmHg}$ [SBP $<130$ and DBP $<80 \mathrm{mmHg}$, respectively, in patients with diabetes]) at week 26 (LOCF) was $49.2 \%$ overall, $49.5 \%$ for aliskiren and $48.3 \%$ for aliskiren HCT (Table 3). When analyzed according to diabetic status, few patients with diabetes $(n=320 ; 16.3 \%)$ achieved a BP goal of <130/80 mmHg; by contrast, $73.1 \%$ $(n=1,977)$ of nondiabetic patients achieved a therapeutic goal of $<140 / 90 \mathrm{mmHg}$. In the additional retrospective analysis, whereby therapeutic goal and BP response criteria were revised with a "classic" BP target of $<140 \mathrm{mmHg}$ and $<90 \mathrm{mmHg}$ and applied to all patients, the proportion of patients achieving the therapeutic goal increased to more than 70\% at week 26 (LOCF; Table 3). Furthermore, no difference was seen with regard to achievement of therapeutic BP goal between patients with diabetes (73.8\%) and the total population (73.4\%).

Both aliskiren and aliskiren HCT were associated with high $\mathrm{BP}$ response rates. At week 26 (LOCF), the proportion of aliskirentreated patients achieving the predefined response for SBP and DBP was $83.6 \%$ and $84.4 \%$, respectively; the corresponding BP response rates in patients receiving aliskiren HCT were $84.4 \%$ and $86.5 \%$. Treatment with aliskiren and aliskiren HCT was also associated with significant reductions from baseline to the end of study in msSBP and msDBP (Fig. 1). At week 26 (LOCF), mean reductions

Table 3 Proportion of patients reaching the predefined therapeutic blood pressure goal and the revised, classic therapeutic blood pressure goal at week 26 (LOCF)

\begin{tabular}{lll}
\hline Treatment & Therapeutic BP goal, $\boldsymbol{n}(\%)$ & “Classic” therapeutic BP goal ${ }^{\mathrm{b}}, \boldsymbol{n}(\%)$ \\
\hline Aliskiren $(n=3,473)^{\mathrm{c}}$ & $1,654(49.5)$ & $2,396(71.7)$ \\
Aliskiren $\mathrm{HCT}(n=1,353)^{\mathrm{c}}$ & $643(48.3)$ & $1,035(77.7)$ \\
All groups combined $(n=4,826)^{\mathrm{c}}$ & $2,297(49.2)$ & $3,431(73.4)$ \\
\hline
\end{tabular}

Full analysis set

$B P$ blood pressure, DBP diastolic blood pressure, $L O C F$ last observation carried forward, HCT hydrochlorothiazide, SBP systolic blood pressure

${ }^{a} \mathrm{SBP} / \mathrm{DBP}<140 / 90 \mathrm{mmHg}(<130 / 80 \mathrm{mmHg}$ for patients with diabetes)

b $\mathrm{SBP} / \mathrm{DBP}<140 / 90 \mathrm{mmHg}$

${ }^{\mathrm{c}}$ The total number of patients with post-baseline value is $n=3,341$ for aliskiren, $n=1,332$ for aliskiren $\mathrm{HCT}$ and $n=4,673$ for all groups combined 


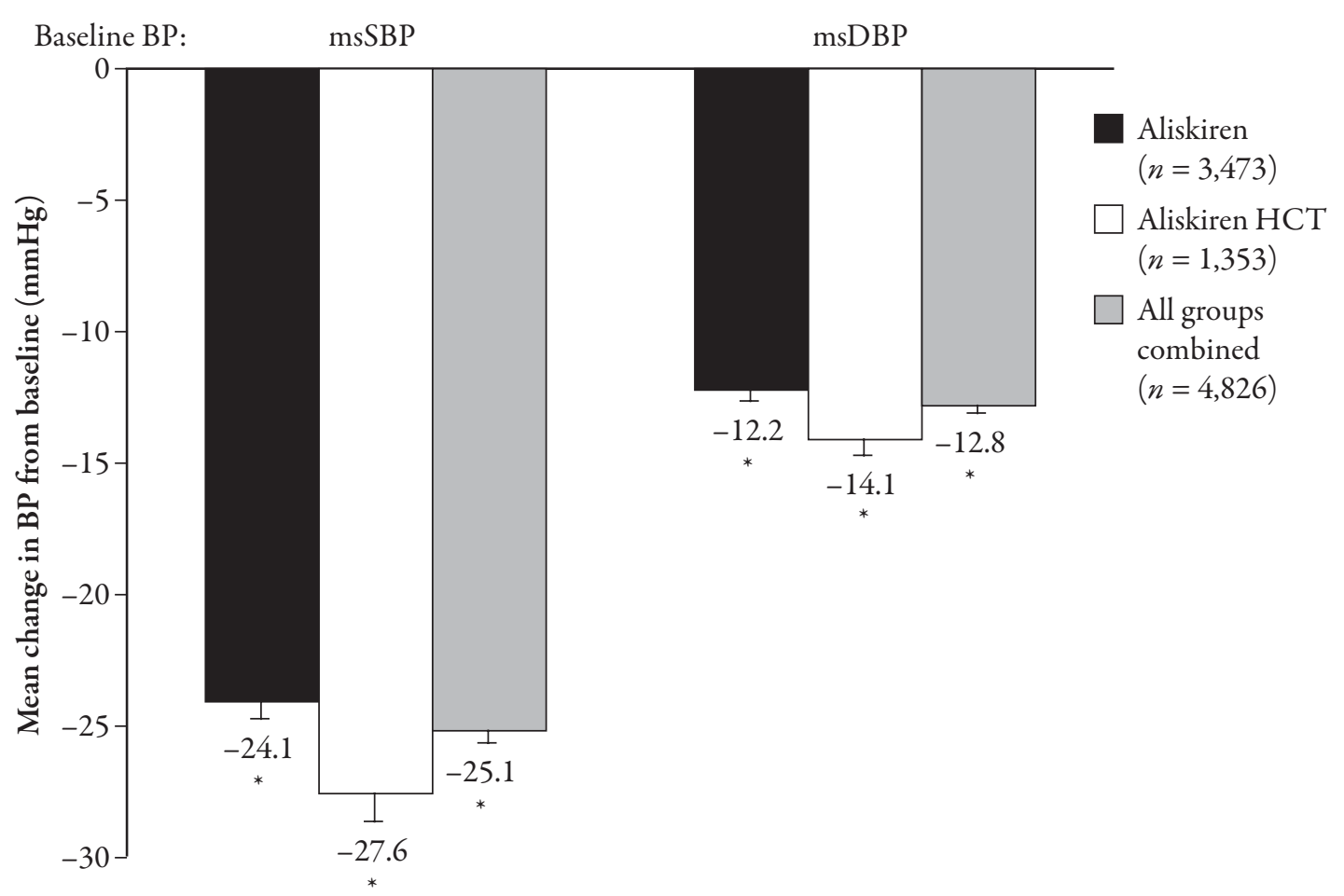

Fig. 1 Mean (standard error) change from baseline to week 26 (last observation carried forward) in mean sitting systolic blood pressure and mean sitting diastolic blood pressure according to treatment group. Full analysis set. $B P$ blood pressure, $H C T$ hydrochlorothiazide, $m s D B P$ mean sitting diastolic blood pressure, $m s S B P$ mean sitting systolic blood pressure. ${ }^{*} P<0.001$ for msSBP and msDBP versus baseline BP

in $\mathrm{msSBP} / \mathrm{msDBP}$ were $24.1 / 12.2 \mathrm{mmHg}$ for aliskiren and 27.6/14.1 $\mathrm{mmHg}$ for aliskiren HCT ( $P<0.001$ vs. baseline for both treatments). Changes in mean sitting BP were numerically greater with increasing severities of baseline BP (Fig. 2). Indeed, in those patients $(n=420)$ with severe hypertension (SBP of $\geq 180 \mathrm{mmHg}$ ), msSBP declined by $49.3 \mathrm{mmHg}$ from baseline $(P<0.001)$. In general, high $\mathrm{BP}$ response rates $(>86 \%)$ as well as consistent reductions in BP compared with baseline (Fig. 3) were observed in important subgroups such as patients with diabetes, the elderly (aged $\geq 65$ years), obese patients (BMI $\geq 30 \mathrm{~kg} / \mathrm{m}^{2}$ ) and those with isolated systolic hypertension (ISH) relative to the overall population. Furthermore, aliskiren and aliskiren HCT were associated with meaningful reductions in BP when prescribed either alone, or as add-on to existing therapy.
At week 26, the msSBP/msDBP change from baseline was $-23.9 /-13.0 \mathrm{mmHg}$ when aliskiren or aliskiren HCT were prescribed alone; the corresponding values when aliskiren or aliskiren HCT were prescribed as add-on therapies were $-26.4 /-12.5 \mathrm{mmHg}$.

\section{Safety and Tolerability}

AEs occurred in a total of 101 (2.1\%) patients (Table 4). The most common AEs included headache, onset of diabetes mellitus, abdominal discomfort, and dizziness (Table 4). The most frequently reported AEs that were suspected to be related to the medication of interest included abdominal discomfort, dizziness, headache, myalgia, and rash. Four deaths were reported during the study; three in the aliskiren group $(0.1 \%)$ and one in the aliskiren HCT group 


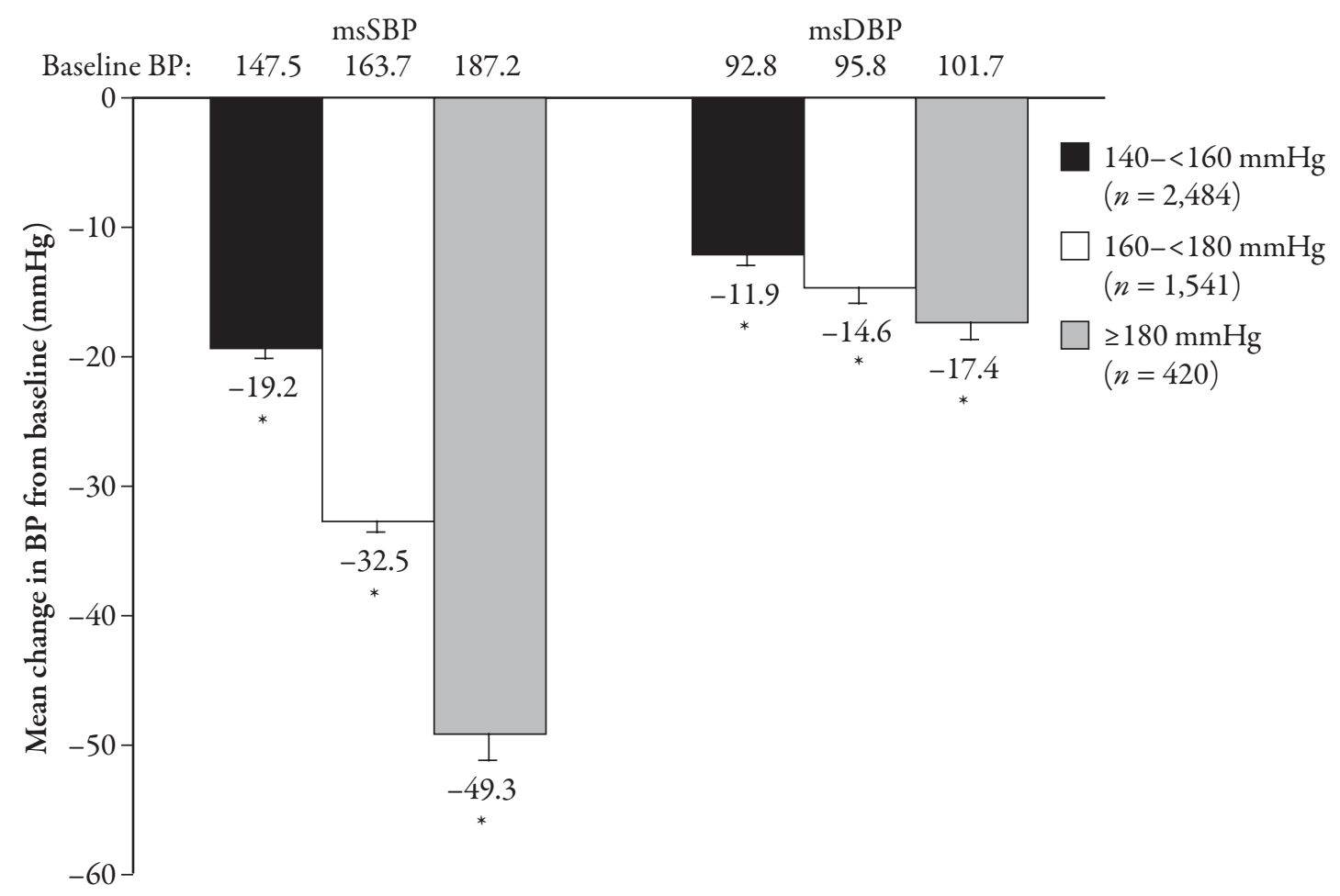

Fig. 2 Mean (standard error) change from baseline to week 26 (last observation carried forward) in mean sitting systolic blood pressure and mean sitting diastolic blood pressure according to severity of hypertension for all groups combined. Full analysis set. $B P$ blood pressure, $m s D B P$ mean sitting diastolic blood pressure, $m s S B P$ mean sitting systolic blood pressure. ${ }^{*} P<0.001$ for $\mathrm{msSBP}$ and $\mathrm{msDBP}$ versus baseline BP

$(0.1 \%)$. None of the deaths were considered to be related to the medication of interest; reasons for death included pneumonia, sepsis, cerebral infarction, and chronic renal failure. Few patients in the overall population reported SAEs $(n=13 ; 0.3 \%)$. The majority of SAEs were considered unrelated to the medication of interest with the exception of five events, which occurred in three patients (angioedema, hypersensitivity, rash, hyperkalemia, increased blood creatinine). Pneumonia was the most common SAE and occurred in three patients. In total, AEs or SAEs led to permanent discontinuation of the medication of interest in 16 patients. Hyperkalemia was observed in 20 patients $(0.8 \%)$, nine of whom were found to have potassium values of $\geq 6.0 \mathrm{mmol} / \mathrm{L}$, while hypokalemia (potassium $<3.5 \mathrm{mmol} / \mathrm{L}$ ) was recorded in 27 patients $(1.1 \%)$ over the course of the study.

\section{Investigator Assessment of Effectiveness, Tolerability, and Treatment Compliance}

At the end of the study, investigators assessed the effectiveness and tolerability of aliskiren and aliskiren HCT, as well as patients' compliance with treatment. Overall, effectiveness of treatment was rated as "very good" or "good" for $89.3 \%$ of all patients, while tolerability was assessed as "very good" or "good" for 93.7\% of patients. Compliance with treatment was rated as "very good" or "good" for $92.5 \%$ of all patients. At the end of the study, treatment with aliskiren or aliskiren HCT was continued by $95 \%$ of all patients. 
$\mathrm{msSBP}$

Baseline BP: $\quad 154.8154 .2153 .2157 .6156 .3$

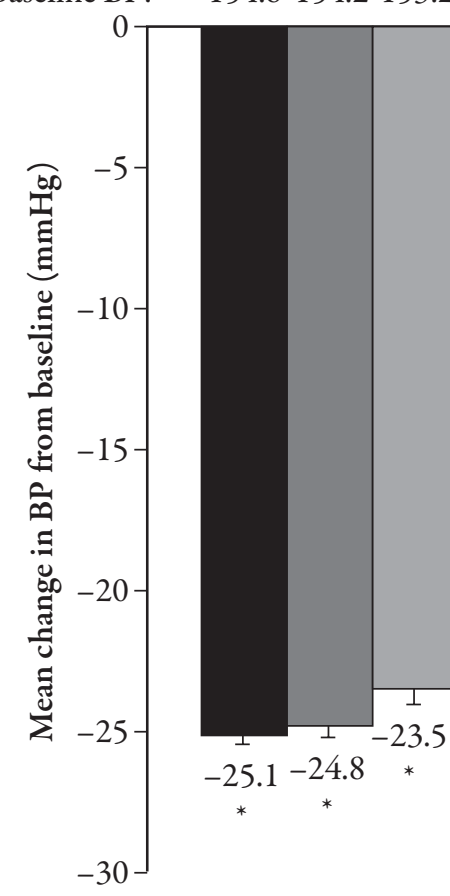

$\mathrm{msDBP}$

$\begin{array}{lllll}93.9 & 92.7 & 81.5 & 90.9 & 94.8\end{array}$

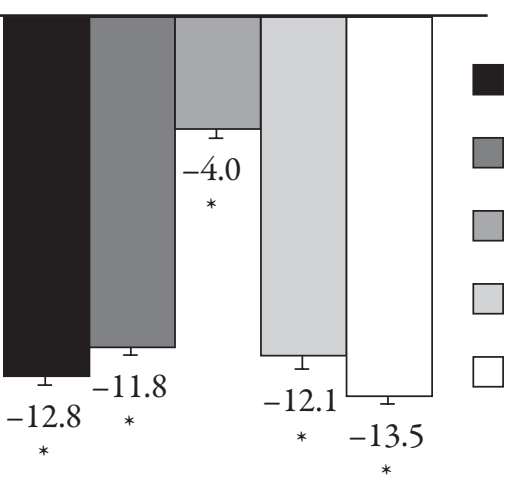

Overall $(n=4,826)$

Diabetes $(n=2,005)$

$\operatorname{ISH}(n=629)$

Elderly $(n=572)$

Obese $(n=1,480)$

Fig. 3 Mean (standard error) change from baseline to week 26 (last observation carried forward) in mean sitting systolic blood pressure and mean sitting diastolic blood pressure according to patient subgroup for all groups combined. Full analysis set. ISH defined as systolic blood pressure $\geq 140 \mathrm{mmHg}$ and diastolic blood pressure $<90 \mathrm{mmHg}$; elderly defined as age $\geq 65$ years; obese defined as body mass index $\geq 30 \mathrm{~kg} / \mathrm{m}^{2}$. BP blood pressure, ISH isolated systolic hypertension, $m s D B P$ mean sitting diastolic blood pressure, $m s S B P$ mean sitting systolic blood pressure.

${ }^{*} P<0.001$ for $\mathrm{msSBP}$ and $\mathrm{msDBP}$ versus baseline $\mathrm{BP}$ for all subgroups

\section{DISCUSSION}

In this real-life study, performed in routine clinical practice in patients from Asia and the Middle East, treatment with aliskiren or aliskiren HCT either as single or add-on therapy resulted in almost half of all patients (49.2\%) with hypertension achieving the prespecified therapeutic BP goal of $<140 / 90 \mathrm{mmHg}$ $(<130 / 80 \mathrm{mmHg}$ in patients with concomitant diabetes). It is noteworthy that 2,005 patients $(41.5 \%)$ in this study had comorbid diabetes mellitus, and when therapeutic BP goal data were analyzed by diabetic status, just $16.3 \%$ of these patients were found to have achieved the more aggressive BP target of
$<130 / 80 \mathrm{mmHg}$, thus impacting the overall therapeutic BP goal. Indeed, when a classic BP target of $<140 / 90 \mathrm{mmHg}$ was applied to all patients, therapeutic BP goal rates increased to $73.4 \%$ in the overall patient population and $73.8 \%$ in patients with diabetes. One of the potential reasons for the low proportion of patients with hypertension and diabetes achieving BP $<130 / 80 \mathrm{mmHg}$ might be due to "therapeutic inertia" among treating physicians, i.e., the failure of physicians to continue to treat to target BP once the classic BP target had been achieved. Therapeutic inertia is a key reason for failure to achieve recommended BP goals [22] and is a common problem in clinical practice worldwide, including countries such as 
Table 4 Most frequently reported adverse events

\begin{tabular}{llll}
\hline Adverse event & Aliskiren & Aliskiren HCT & Total \\
& $n=3,473=4,826$ & $n(\%)$ & $n(\%)$ \\
& $n(\%)$ & $28(2.1)$ & $101(2.1)$ \\
\hline Any & $73(2.1)$ & $1(0.1)$ & $8(0.2)$ \\
Headache & $7(0.2)$ & $5(0.4)$ & $6(0.1)$ \\
Onset of diabetes mellitus & $1(0.0)$ & $0(0.0)$ & $5(0.1)$ \\
Abdominal discomfort & $5(0.1)$ & $0(0.0)$ & $5(0.1)$ \\
Dizziness & $5(0.1)$ & $1(0.1)$ & $4(0.1)$ \\
Diarrhea & $3(0.1)$ & $0(0.0)$ & $4(0.1)$ \\
Myalgia & $4(0.1)$ & $0(0.0)$ & $4(0.1)$ \\
Rash & $4(0.1)$ & $3(0.2)$ & $4(0.1)$ \\
Hypertension & $1(0.0)$ & $0(0.0)$ & $3(0.1)$ \\
Asthenia & $3(0.1)$ & $0(0.0)$ & $3(0.1)$ \\
Pneumonia & $3(0.1)$ & $1(0.1)$ & $3(0.1)$ \\
Weight increased & $2(0.1)$ & $3(0.2)$ & $3(0.1)$ \\
Hyperglycemia & $0(0.0)$ & $2(0.1)$ & $3(0.1)$ \\
Hyperuricemia & $1(0.0)$ & $0(0.0)$ & $3(0.1)$ \\
Cough & $3(0.1)$ & $0(0.0)$ & $3(0.1)$ \\
Hypotension & $3(0.1)$ & & $n$
\end{tabular}

Full analysis set

HCT hydrochlorothiazide

Pakistan [23] and the United Arab Emirates [24] where under-diagnosis and under-treatment rates pose serious challenges.

The question of optimal BP goals in different groups of patients with hypertension has long been a subject of intense debate. In particular, the lower BP thresholds for patients with complicated hypertension, e.g., diabetes or renal disease, have come under scrutiny in recent years as a result of studies such as Action to Control Cardiovascular Risk in Diabetes (ACCORD). This trial showed that there was no benefit to be gained in a high-risk patient population with diabetes from intensively lowering SBP to a goal of $<120 \mathrm{mmHg}$, with no difference reported in the primary endpoint (a composite of fatal and nonfatal major CV events) between this group and those who received standard therapy to lower BP to $<140 \mathrm{mmHg}$ [25].
Furthermore, the reappraisal of the European hypertension guidelines proposes that the BP goal for all patients with hypertension is set to $<140 / 90 \mathrm{mmHg}$ regardless of comorbidities or level of CV risk [26]. The authors of this reappraisal argue that the traditionally recommended BP goal of $<130 / 80 \mathrm{mmHg}$ in hypertensive patients with comorbid diabetes is not thought to be supported by clinical trial outcome evidence, nor is it thought to be a realistic target [26]. Similarly, a panel assembled by the Kidney Disease Improving Global Outcomes (KDIGO) recently raised the BP target from $<130 / 80 \mathrm{mmHg}$ to $<140 / 90 \mathrm{mmHg}$ in patients with chronic kidney disease, including those with proteinuria, based on evidence from a number of large clinical trials [27-29].

The present study also showed that during the observation and treatment period of 
$26 \pm 8$ weeks, both aliskiren and aliskiren HCT produced significant reductions from baseline in msSBP and msDBP (25.1 mmHg and $12.8 \mathrm{mmHg}$, respectively; both $P<0.001$ vs. baseline). The magnitude of reduction in BP was dependent on the baseline value, such that in patients with severe hypertension (SBP of $\geq 180 \mathrm{mmHg}$ ), reductions of $49.3 \mathrm{mmHg}$ and $17.4 \mathrm{mmHg}$ in msSBP and msDBP, respectively, were recorded at the end of the study. Similar reductions in BP to the overall results were also reported for the various patient subgroups, including patients with diabetes or ISH, and those who were obese or aged $\geq 65$ years. Moreover, BP responder rates were consistently achieved in more than $80 \%$ of all patients during the study.

In general, the results observed in this realworld, multiethnic study appear to correspond broadly with the results seen in other randomized, controlled trials of aliskiren and/or aliskiren HCT in different patient populations with hypertension, including those with metabolic comorbidities, such as diabetes or obesity [30], and the elderly [17]. The results of the present study are also in line with those reported in a recent observational, multicenter, openlabel study conducted in 2,070 patients with hypertension (25.3\% of whom were also diabetic) in a "real-world" setting in Belgium [18]. In this particular study, mean reductions in SBP/DBP at 180 days following treatment with aliskiren $300 \mathrm{mg}$ were 22.9 and $10.5 \mathrm{mmHg}$, respectively ( $P<0.001$ vs. baseline for both). BP control (defined as $<140 / 90 \mathrm{mmHg}$ or $<130 / 80 \mathrm{mmHg}$ in patients with diabetes) was achieved in $36.4 \%$ of patients, and this increased to $56.3 \%$ when the 2009 European guidelines criteria were applied, i.e., 130-139/80-89 mmHg. Other observational trials, specifically the $3 \mathrm{~A}$ registry of 14,988 patients in Germany [20] and the Aliskiren Canadian Hypertension Registry (ANCHOR) of approximately 15,000 patients with hypertension with or without type 2 diabetes [19], should help to provide further important information regarding the use and effectiveness of aliskiren in real-life practice conditions.

As with many observational studies, the reported incidence of AEs was low (2.1\%). Although the most frequently reported AEs were headache, onset of diabetes mellitus, abdominal discomfort, and dizziness, each event was reported in less than $0.2 \%$ of all patients. The four deaths that occurred during the observational period, as well as the majority of SAEs, were not considered to be study-drug related. Recognizing the inherent limitations with observational studies, aliskiren-based therapy appeared to be well tolerated in this hypertensive population, with a profile not dissimilar to what has been reported in other randomized, controlled trials [14, 17, 30].

We note the findings of the recently presented Aliskiren Trial In Type 2 Diabetes Using CardioRenal Disease Endpoints (ALTITUDE) study, where no benefit of a dual RAAS regimen (including aliskiren) was observed, and a greater incidence of AEs was noted in patients with type 2 diabetes and renal impairment compared with standard of care over a median follow-up period of 32.9 months [31]. We conducted a posthoc subanalysis of the hypertensive patients with diabetes in the present study who had received a dual RAAS regimen; in the present study population the overall incidence of AEs was $1.3 \%(8 / 607)$ in the diabetic patients who received dual RAAS, compared with an incidence of $2.2 \%(93 / 4,219)$ in all other patients in the study. These data, of course, should be viewed in the context of the observational nature of the study, the different study population recruited, and the shorter treatment duration. Consequently, despite the benign safety data observed in the present study, we would still caution against the use of aliskiren in a diabetic 
population as add-on to another RAAS inhibitor, in line with the updated prescribing information available from the study sponsor.

An additional element of the present study was the investigator assessment of treatment effectiveness, tolerability, and compliance. Overall, the effectiveness and tolerability of aliskiren and aliskiren HCT was rated as "good" or "very good" for almost all patients (effectiveness: 89.3\%; tolerability: $93.7 \%)$. Treatment compliance was also judged to be "good" or "very good" for more than $90 \%$ of patients; a reflection of the proportion of patients (95\%) who continued on treatment after the study. Accepting that heightened compliance may partly be a consequence of patient involvement in a study, these findings are noteworthy nonetheless given that noncompliance with treatment is a major obstacle to achieving BP goal worldwide, including South and East Asia where challenges surrounding economic burden and access to treatment dominate [32, 33].

A potential limitation of this study is its nonrandomized, open-label, single-arm design, which may allow for selection bias or confounding variables, so potentially limiting the interpretation of results. However, this design was adopted to reflect the effectiveness, tolerability, and safety of aliskiren and aliskiren HCT in daily clinical practice. Furthermore, loss to follow-up is typically seen as a drawback of observational studies, although the present results indicated that only a small proportion of patients were lost to follow-up (3.8\%). The subjective evaluation of the effectiveness, tolerability, and compliance with aliskiren and aliskiren HCT is also a limitation of this study. Nevertheless, the observational nature of this trial allowed for the collection of a substantial amount of data from a multiethnic, hypertensive patient population where aliskiren and aliskiren HCT were prescribed in a naturalistic setting.

\section{CONCLUSIONS}

Treatment with either aliskiren or aliskiren HCT alone or as add-on therapy was associated with statistically significant and clinically meaningful reductions in BP compared with baseline. Almost half of all patients achieved a therapeutic BP goal of $<140 / 90$ (and $<130 / 80 \mathrm{mmHg}$ in patients with diabetes) and this number increased to in excess of $70 \%$ when a therapeutic BP goal of $<140 / 90 \mathrm{mmHg}$ was applied to all patients. In this real-world, naturalistic setting, treatment based on aliskiren and aliskiren HCT proved to be effective and well-tolerated BP-lowering therapies in this multiethnic population with arterial hypertension.

\section{ACKNOWLEDGMENTS}

The authors acknowledge all investigators at the participating centers and all patients for their commitment to the study, which was supported by Novartis Pharma AG, Basel, Switzerland. The authors also acknowledge Birgit Schmidtmann, Alexander Pieper and Anja Schade, DATAMAP GmbH, for data collection and data analysis.

All authors participated in the development and writing of the paper and took full responsibility for the content of the paper. The authors were assisted in the preparation of this text by professional medical writer, Sharon Smalley, at CircleScience, Macclesfield, UK; this support was funded by Novartis Pharma AG.

Dr. S. R. Maddury is the guarantor for this article, and takes responsibility for the integrity of the work as a whole.

This study was presented as a poster presentation at the $27^{\text {th }}$ Annual Scientific Meeting and Exposition of the American Society of Hypertension, New York, NY, USA, May 19-22, 2012. 
Conflict of interest. Dr. S. R. Maddury has received an honorarium and has a consultancy agreement with Novartis Healthcare Private Limited for the conduct of the trial. Dr. A. Pande has received an honorarium from Novartis Healthcare Private Limited. Dr. K. M. H. S. S. Haque has received an honorarium and has a consultancy agreement with Novartis Bangladesh Limited for the conduct of the trial. Dr. A. Echtay has received honoraria for speaking engagements for Novartis, MSD, Sanofi, Lilly, Pfizer, Servier, Merck, Novo Nordisk, AstraZeneca and Menarini. Dr. T. Gulzar has received an honorarium and has a consultancy agreement with Novartis Healthcare Private Limited for the conduct of the PMS trial. Dr. L. Go has received an honorarium and has a consultancy agreement with Novartis Healthcare Philippines Inc. for the conduct of this trial. Drs. M. Kadwa and S. Hristoskova are employees of Novartis Pharma AG and are eligible for stock and stock options; both employees currently hold shares in Novartis Pharma AG. The page charges for this paper were funded by Novartis Pharma AG.

\section{REFERENCES}

1. Ezzati M, Lopez AD, Rodgers A, Vander Hoorn S, Murray CJ; Comparative Risk Assessment Collaborating Group. Selected major risk factors and global and regional burden of disease. Lancet. 2002;360:1347-60.

2. Kearney PM, Whelton M, Reynolds K, Muntner P, Whelton PK, He J. Global burden of hypertension: analysis of worldwide data. Lancet. 2005;365: 217-23.

3. Chobanian AV, Bakris GL, Black HR, et al. The seventh report of the Joint National Committee on Prevention, Detection, Evaluation, and Treatment of High Blood Pressure. JAMA. 2003;289:2560-72.

4. Kannel WB, D'Agostino RB, Cobb JL. Effect of weight on cardiovascular disease. Am J Clin Nutr 1996;63(3 Suppl.):419S-22S.
5. Kannel WB. Fifty years of Framingham Study contributions to understanding hypertension. J Hum Hypertens. 2000;14:83-90.

6. Lawes CM, Vander Hoorn S, Rodgers A; International Society of Hypertension. Global burden of blood-pressure-related disease, 2001. Lancet. 2008;371:1513-8.

7. Lewington S, Clarke R, Qizilbash N, Peto R, Collins $\mathrm{R}$; Prospective Studies Collaboration. Age-specific relevance of usual blood pressure to vascular mortality: a meta-analysis of individual data for one million adults in 61 prospective studies. Lancet. 2002;360:1903-13.

8. Wolf-Maier K, Cooper RS, Kramer H, et al. Hypertension treatment and control in five European counties, Canada, and the United States. Hypertension. 2004;43:10-7.

9. Antikainen RL, Moltchanov VA, Chukwuma C $\mathrm{Sr}$, et al. Trends in the prevalence, awareness, treatment and control of hypertension: the WHO MONICA Project. Eur J Cardiovasc Prev Rehabil. 2006;13:13-29.

10. Sison J, Arceo LP, Trinidad E, et al. Philippine Heart Association-Council on Hypertension Report on survey of hypertension and target organ damage (PRESYON 2-TOD). A report on prevalence of hypertension, awareness, treatment profile and control rate. Philipp J Cardiol. 2007;35:1-9.

11. Burnier M, Brown RE, Ong SH, Keskinaslan A, Khan ZM. Issues in blood pressure control and the potential role of single-pill combination therapies. Int J Clin Pract. 2009;63:790-8.

12. Azizi M, Webb R, Nussberger J, Hollenberg NK. Renin inhibition with aliskiren: where are we now, and where are we going? J Hypertens. 2006;24:24356.

13. Müller DN, Luft FC. Direct renin inhibition with aliskiren in hypertension and target organ damage. Clin J Am Soc Nephrol. 2006;1:221-8.

14. Black HR, Kribben A, Aguirre Palacios F, Bijarnia M, Laflamme AK, Baschiera F. Aliskiren alone or in combination with hydrochlorothiazide in patients with the lower ranges of stage 2 hypertension: The ACQUIRE randomized double-blind study. J Clin Hypertens (Greenwich). 2010;12:917-26.

15. Krone W, Hanefeld M, Meyer HF, et al. Comparative efficacy and safety of aliskiren and irbesartan in patients with hypertension and metabolic syndrome. J Hum Hypertens. 2011;25:186-95. 
16. Whaley-Connell A, Purkayastha D, Yadao A, Sowers JR. Central pressure and biomarker responses to renin inhibition with hydrochlorothiazide and ramipril in obese hypertensives: the ATTAIN study. Cardiorenal Med. 2011;1:53-66.

17. Duprez DA, Munger MA, Botha J, Keefe DL, Charney AN. Aliskiren for geriatric lowering of systolic hypertension: a randomized controlled trial. J Hum Hypertens. 2010;24:600-8.

18. Verpooten GA, Aerts A, Coen $\mathrm{N}$, et al. Antihypertensive effectiveness of aliskiren for the "real-world" management of hypertension: multilevel modelling of 180-day blood pressure outcomes (the Belgian DRIVER Study). Int J Clin Pract. 2011;65:54-63.

19. De Cotret P, Steele A, Leclerc JM, et al. Reallife blood pressure reductions in hypertension patients treated with aliskiren. Interim results of the Canadian Anchor registry (4A.05). J Hypertens. 2011(eSuppl. A);29:e48.

20. Zeymer U, Dechend R, Deeg E, et al. Aliskiren for the treatment of essential hypertension under real-life practice conditions: design and baseline data of the prospective $3 \mathrm{~A}$ registry. Int J Clin Pract. 2012;66:251-61.

21. American Heart Association Committee. American Heart Association Committee report on blood pressure determination. Hypertension. 1988;1210A-22A.

22. Düsing R. Overcoming barriers to effective blood pressure control in patients with hypertension. Curr Med Res Opin. 2006;22:1545-53.

23. Saleem F, Hassali AA, Shafie AA. Hypertension in Pakistan: time to take some serious action. Br J Gen Pract. 2010;60:449-50 [Letter].

24. Abdulle AM, Nagelkerke NJ, Abouchacra S, Pathan JY, Adem A, Obineche EN. Under-treatment and under diagnosis of hypertension: a serious problem in the United Arab Emirates. BMC Cardiovasc Disord. 2006;6:24.
25. ACCORD Study Group, Cushman WC, Evans $\mathrm{GW}$, et al. Effects of intensive blood-pressure control in type 2 diabetes mellitus. N Engl J Med. 2010;362:1575-85.

26. Mancia G, Laurent S, Agabiti-Rosei E, et al. Reappraisal of European guidelines on hypertension management: a European Society of Hypertension Task Force document. J Hypertens. 2009;27:2121-58.

27. Ruggenenti P, Perna A, Loriga G, et al. Bloodpressure control for renoprotection in patients with non-diabetic chronic renal disease (REIN-2): multicentre, randomised controlled trial. Lancet. 2005;365:939-46.

28. Wright JT Jr., Bakris G, Greene T, et al. Effect of blood pressure lowering and antihypertensive drug class on progression of hypertensive kidney disease: results from the AASK trial. JAMA. 2002;288:2421-31.

29. Peralta CA, Norris KC, Li S, et al. Blood pressure components and end-stage renal disease in persons with chronic kidney disease: the Kidney Early Evaluation Program (KEEP). Arch Intern Med. 2012;172:41-7.

30. Andersen $\mathrm{K}$, Weinberger $\mathrm{MH}$, Egan $\mathrm{B}$, et al. Comparative efficacy and safety of aliskiren, an oral direct renin inhibitor, and ramipril in hypertension: a 6-month, randomized, doubleblind trial. J Hypertens. 2008;26:589-99.

31. Parving $\mathrm{HH}$, Brenner BM, McMurray JJ, et al. Cardiorenal end points in a trial of aliskiren for type 2 diabetes. N Engl J Med. 2012;367:2204-13.

32. Hussain SM, Boonshuyar C, Ekram ARMS. Nonadherence to antihypertensive treatment in essential hypertensive patients in Rajshahi, Bangladesh. AKMMC J. 2011;2:9-14.

33. Wagner AK, Valera M, Graves AJ, Laviña S, RossDegnan D. Costs of hospital care for hypertension in an insured population without an outpatient medicines benefit: an observational study in the Philippines. BMC Health Serv Res. 2008;8:161. 